\title{
Importance of trabecular anisotropy in finite element predictions of patellar strain after Total Knee Arthroplasty
}

\author{
A. Latypova, D.P. Pioletti, A. Terrier* \\ Laboratory of Biomechanical Orthopedics, Ecole Polytechnique Fédérale de Lausanne (EPFL), Station 19, 1015 Lausanne, Switzerland
}

\section{A R T I C L E I N F O}

\section{Article history:}

Received 13 August 2015

Revised 19 October 2016

Accepted 23 October 2016

Available online xxx

\section{Keywords:}

Patella

Bone mechanics

Anisotropy

Finite element analysis

Arthroplasty

\begin{abstract}
A B S T R A C T
Patellar fracture and anterior knee pain remain major complications after Total Knee Arthroplasty (TKA). Patient-specific finite element (FE) models should help improve understanding of these complications through estimation of joint and bone mechanics. However, sensitivity of predictions on modeling techniques and approaches is not fully investigated. In particular, the importance of patellar bone anisotropy, usually omitted in FE models, on strain prediction is still unknown. The objective of this study was thus to estimate the influence of modeling patellar trabecular anisotropy on prediction of patellar strain in TKA models.

We compared FE-derived strain predictions with isotopic and anisotropic material properties using 17 validated FE models of the patella after TKA. We considered both non-resurfaced and resurfaced patellae, in a load-bearing TKA joint. We evaluated and compared the bone volume above a strain threshold and, in addition, estimated if the difference in isotopic and anisotropic predictions was consistent between patellae of different average bone volume fraction.

Compared to the anisotropic reference, the isotropic prediction of strained volume was $3.7 \pm 1.8$ times higher for non-resurfaced patellae and $1.5 \pm 0.4$ times for resurfaced patellae. This difference was higher for patellae with lower average bone volume fraction.

This study indicates that strain predictions acquired via isotropic patellar FE models should be interpreted with caution, especially when patellae of different average bone volume fraction are compared.
\end{abstract}

(c) 2016 IPEM. Published by Elsevier Ltd. All rights reserved.

\section{Introduction}

Patellar fracture and anterior knee pain (AKP) remain one of the major complications after Total Knee Arthroplasty (TKA) [1,2]. It is believed that patellar resurfacing can decrease the risk of AKP, while non-resurfacing can help to avoid fracture and other complications associated with resurfacing [2]. These two surgical procedures are widely used in clinical practice and often compared, but no clear advantage of one over the other is reported [2].

Numerical studies suggest that estimation of patellar strain after TKA could help to better understand its pathologies and to choose an appropriate surgical technique [3-5]. However, literature is lacking a validated patellar material law for accurate strain predictions. Currently, the existing patellar numerical homogenized models (hFE) rely on isotropic material laws obtained from other anatomical sites, such as femora or vertebra $[3,5,6]$

In a previous study, we identified and validated a patellar material law based on morphology-elasticity relationship by means

\footnotetext{
* Corresponding author.

E-mail address: alexandre.terrier@epfl.ch (A. Terrier).
}

of micro-finite element ( $\mu \mathrm{FE})$ modeling of 20 fresh-frozen cadaveric patellae [7]. We considered two alternative models: isotropic and anisotropic. It was shown that the anisotropic model better replicates the $\mu \mathrm{FE}$ reference. The isotropic model underestimated the stiffness of the patella, and thus tended to overestimate bone strain. However, the validation in that study was conducted on the cuboid patellar section by means of tension and shear load testing applied on the sides of the cuboid. To estimate if the isotropy simplification indeed increases strain prediction in clinical applications, the isotropic model should be compared to the anisotropic model during physiological loading conditions. Although there is a potential to measure anisotropy with standard preoperative computed tomography (CT) scans [8], this modeling approach has not yet been validated. Thus, the isotropic model has higher potential to be used in clinical applications. The estimation of influence of the isotropic simplification on strain prediction is therefore of great importance.

Hypothesizing that anisotropy plays a crucial role in patellar strain prediction, the aim of this study was to compare calculated patellar strains during a loaded knee flexion after TKA using isotropic and anisotropic validated models. We considered both non-resurfaced and resurfaced patella, since these two cases are 
often compared. In addition, we evaluated if the difference in isotopic and anisotropic strain predictions is consistent between patellae of different average bone volume fraction, since it has been suggested that predictions of isotropic models of bones with low bone volume fraction will be higher deviated from anisotropic models predictions [9].

\section{Materials and methods}

Seventeen fresh-frozen cadaveric patellae (10 males, 7 females; age range $34-93$, mean age $70 \pm 18$ ) were used for the study. The strains of each patella were evaluated by an isotropic and an anisotropic validated hFE model [7], in a non-resurfaced and resurfaced patella option of TKA. The applied boundary conditions were provided by a validated musculoskeletal knee model [10]. The effect of the isotropic simplification was estimated by comparing the strain predictions with the anisotropic reference.

The model included the patella, the cartilage (non-resurfaced), the patellar component (resurfaced), the surface of the femoral component, the patellar ligament and the four quadriceps muscles: vastus lateralis (VL), vastus medialis (VM), rectus femoris (RF) and vastus intermedius (VI). The geometry of each patella was extracted from segmented $\mu \mathrm{CT}$ scans, obtained during a previous study [7], and imported to Geomagic (Geomagic, Inc., Morrisville, North Carolina, USA) to create non-uniform rational Bspline (NURBS) surfaces. To simulate non-resurfaced cases, we added a cartilage layer for each patella by a uniform extrusion $(3 \mathrm{~mm})$ of the posterior articular surface of the patellar bone [6]. To simulate the resurfaced cases, we replicated recommendations of the manufacturer (Symbios, Yverdon-les-Bain, Switzerland). The posterior part of the patella was cut, three cylindrical holes were removed, and the three-peg modified dome patellar component was inserted [2]. The cut depth (5-9 mm), as well as the prosthetic component size (thickness/diameter: $8 \mathrm{~mm} / 31.2 \mathrm{~mm}$, $8.5 \mathrm{~mm} / 34.2 \mathrm{~mm}, 9 \mathrm{~mm} / 37.2 \mathrm{~mm}$ ), depended on the patellar size and were aimed to preserve the original thickness of the patella without exceeding critical remaining bone thickness of $12 \mathrm{~mm}$. Only the articular surface of the femoral component was included into the model. The geometry of the femoral and patellar components was obtained from the manufacturer. For simplicity, the cement layer was not modeled. We used CAD software Solidworks (Dassault Systèmes, Vélizy, France) to create the cartilage layer, and to cut the bone and position the patellar component.

We replicated a loaded squat at 60 degrees of knee flexion. The position of the femur and tibia was fixed and imposed by the squat movement. The muscle forces were estimated by a validated musculoskeletal TKA model, assuming a constant body weight $(800 \mathrm{~N})$ [10]. The muscle forces (RF: $544 \mathrm{~N}, \mathrm{VI}: 706 \mathrm{~N}, \mathrm{VL}: 1216 \mathrm{~N}, \mathrm{VM}$ : $778 \mathrm{~N}$ ) were distributed according to muscles physiological crosssectional areas [11]. The cartilage (non-resurfaced) and patellar component (resurfaced) were in contact with the surface of the femoral component. The position of the patella was thus only constrained by its contact with the femoral component, the applied muscle forces, and the patellar ligament reaction (Fig. 1).

The Zysset-Curnier morphology-elasticity relationship was considered for the patellar bone [12,13]:

$$
\begin{gathered}
E_{i}=E_{0} \rho^{k}\left(m_{i}^{2}\right)^{l}, \frac{E_{i}}{v_{i j}}=\frac{E_{0}}{v_{0}} \rho^{k}\left(m_{i} m_{j}\right)^{l}, \\
G_{i j}=G_{0} \rho^{k}\left(m_{i} m_{j}\right)^{l}, \forall i \neq j=1,2,3
\end{gathered}
$$

where $E_{i}, v_{i j}$, and $G_{i j}$ are engineering constants, $E_{0}, v_{0}, G_{0}, k, l$ are model parameters, $\rho$ is the bone volume fraction, and $m_{i}$ are the normalized eigenvalues of the fabric tensor $\boldsymbol{M}$ [14]. In the isotropic case the fabric tensor $\boldsymbol{M}$ was equal to the identity tensor $\boldsymbol{I}$. Model
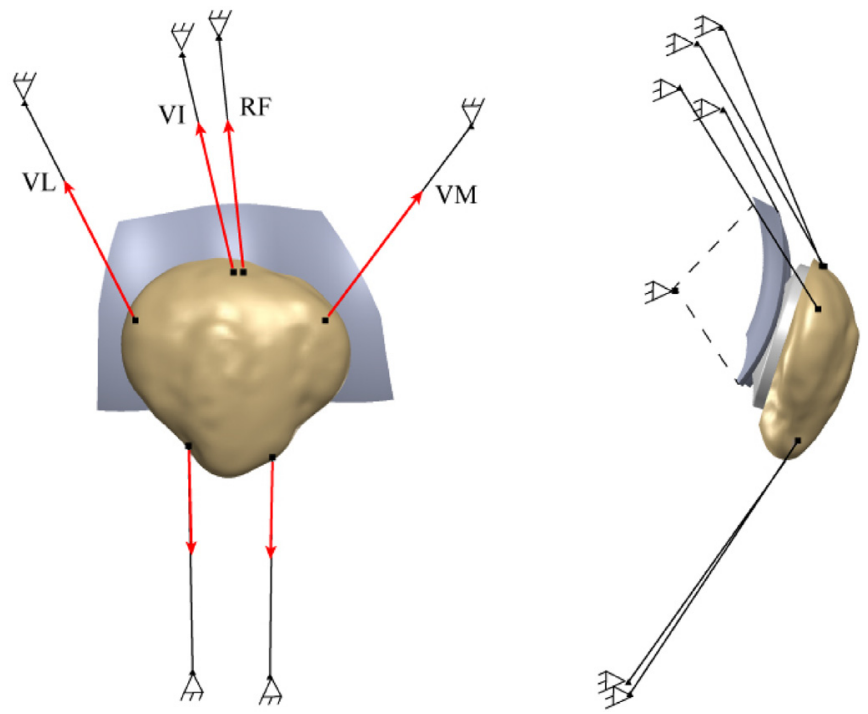

Fig. 1. Patellofemoral TKA model at 60 degrees of knee flexion.

Table 1

Model parameters for isotropic and anisotropic laws.

\begin{tabular}{llllll}
\hline Law & $E_{0}(\mathrm{MPa})$ & $v_{0}$ & $G_{0}(\mathrm{MPa})$ & $k$ & $l$ \\
\hline Isotropic & 11035.98 & 0.26 & 4395.05 & 2.13 & - \\
Anisotropic & 12723.05 & 0.24 & 4224.62 & 2.1 & 1.02 \\
\hline
\end{tabular}

parameters were identified and validated using micro finite element ( $\mu \mathrm{FE})$ modeling on 20 cadaveric patellae (Table 1) [7] .

In the homogenized isotropic and anisotropic models, the material properties of each bone element were assigned from $\mu \mathrm{CT}$ images using Medtool software (www.dr-pahr.at). A background grid with cubic hex elements $(2.0 \mathrm{~mm}$ side length) was defined over $\mu \mathrm{CT}$ data set. A spherical volume with $5.3 \mathrm{~mm}$ diameter was centered at each node of the grid. Bone volume fraction (bone volume over tissue volume) and mean intercept length (MIL) based fabric tensor were computed for each volume and assigned to the node of the grid. Bone volume fraction and fabric tensor were interpolated to the elements of the patellar bone mesh, providing engineering constants and material orientations for all elements. The cortical bone was not modeled explicitly. Cartilage was assumed Neo-Hookean hyperelastic $\left(C_{10}=2 \mathrm{MPa}, k=40 \mathrm{MPa}\right.$, derived from $E=12 \mathrm{MPa}, v=0.45)$ [15], polyethylene was assumed linear elastic $(E=572 \mathrm{MPa}, v=0.4)$ [3]. The femoral component was rigid. The patellar ligament was modeled by two rigid bars.

The model was implemented in Abaqus v6.13 (Simulia, Providence, RI, USA). Patellar bone and patellar component were meshed with linear tetrahedral elements $(2 \mathrm{~mm}$ and $1.6 \mathrm{~mm}$ element size respectively), while cartilage was meshed with linear hexahedral elements ( $1.8 \mathrm{~mm}$ element size). Bone mesh type and size was done based on previous experience [16]. The muscle and ligament forces were distributed along all nodes of the entire anterior patellar surface with cubic weight function [17]. The system contained around $10^{4}$ degrees of freedom. The implicit solver was used.

We evaluated octahedral shear strain of all patellae [18], for the isotropic and anisotropic models, and for the non-resurfaced and resurfaced cases. To compare isotropy to anisotropy, we calculated for the two cases the volume of bone with a strain above a threshold value. The anisotropic case was used as a reference. Three bone volumes of $2 \%, 5 \%$ and $10 \%$ with highest strains in anisotropic case were associated to three stain thresholds. The strain thresholds 


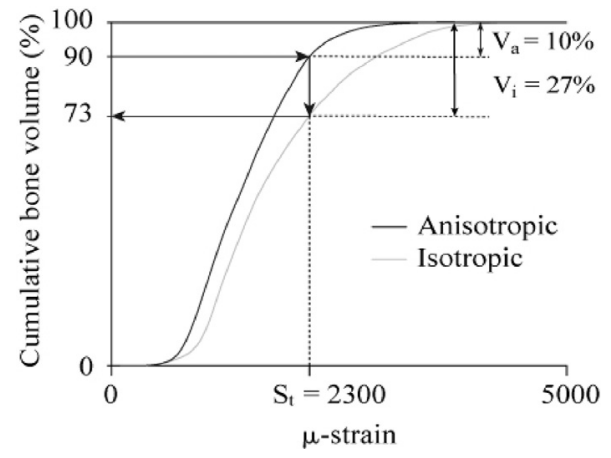

Fig. 2. To compare isotropic and anisotropic models, a reference volume of highly strained bone in anisotropic case $V_{a}$ was associated to a strain threshold $S_{t}$, which was then associated to the comparable bone volume in the isotropic case $V_{i}$. This process is illustrated on the cumulative strain distribution with a reference volume $V_{a}$ of $10 \%$.

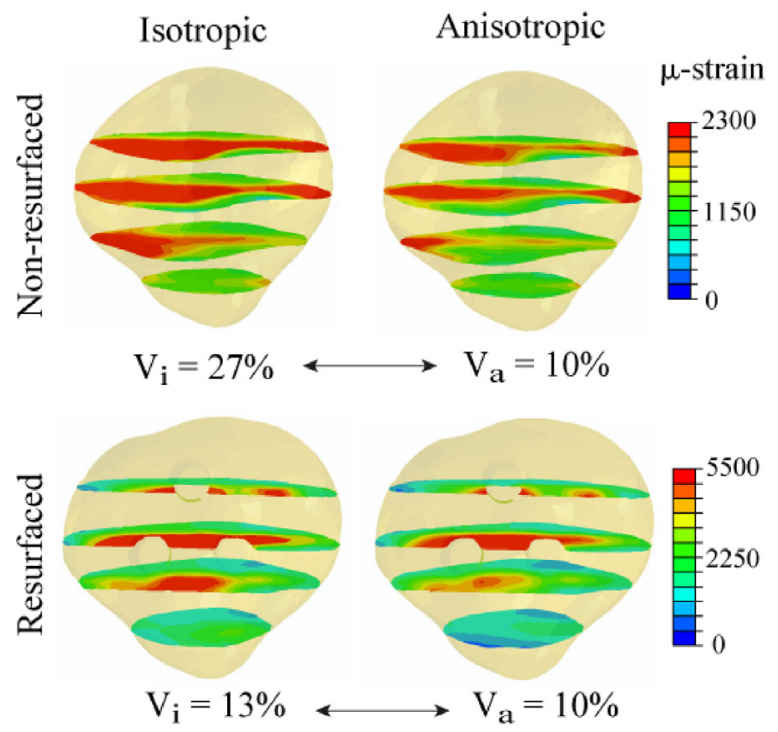

Fig. 3. Strain distribution and bone volumes above the same strain threshold (corresponds to $10 \%$ of bone volume in anisotropic case) predicted by isotropic $\left(V_{i}\right)$ and anisotropic $\left(V_{a}\right)$ models for a non-resurfaced and resurfaced patella.

were thus specific to each patella, and different for the resurfaced and non-resurfaced case. For each strain threshold, we evaluated the corresponding bone volume for the isotropic model (Fig. 2), and normalized it to the volume of the anisotropic reference. In addition, we evaluated the correlation between the average bone volume fraction $\rho$ and the normalized isotropic strained bone volume. The average bone volume fraction was calculated on the entire patellar bone, and was thus different for each patella, and for the resurfaced and non-resurfaced case.

\section{Results}

With the reference anisotropic model, the strain thresholds corresponding to $2 \%, 5 \%$ and $10 \%$ of bone volume, were $5000 \pm 2900$, $4400 \pm 2500,3900 \pm 2100 \mu$ strains for non-resurfaced patellae, and $12100 \pm 7000,8500 \pm 4600,6500 \pm 3550$ for resurfaced patellae. For all resurfaced and non-resurfaced patellae, the isotropic model predicted higher average strains and larger strained bone volumes than the anisotropic model (Fig. 3). For the non-resurfaced patella, the isotropic model predicted $11.1 \pm 3.9 \%, 16.8 \pm 3.8 \%$ and $22.9 \pm 4.5 \%$ of strained bone volume, against the $2 \%, 5 \%$, and $10 \%$ of reference strained bone volume for the anisotropic model. Thus, the isotropic model overestimated strained bone volume by $3.7 \pm 1.8$ times, on average. In the resurfaced case, the difference was less pronounced. The isotropic model predicted $3.4 \pm 1.2 \%$, $7.5 \pm 1.6 \%$ and $14.0 \pm 2.3 \%$ of bone volume, against the $2 \%, 5 \%$, and $10 \%$ of reference bone volume. Thus, the isotropic model overestimated the highly strained bone volume by only $1.5 \pm 0.4$ times, on average.

The average bone volume fraction $\rho$ of the patellae was $0.44 \pm 0.10$ (range 0.27-0.59) for non-resurfaced cases, and $0.46 \pm 0.11$ (range $0.27-0.63$ ) for resurfaced cases. There was a negative correlation between $\rho$ and the normalized isotropic bone strained volume. This correlation depended on the threshold limits. The regression coefficient increased with the increase of bone volume threshold. For the non-resurfaced case, the correlation was significant for $5 \%(r=-0.57, p=0.015)$ and $10 \%(r=-0.64$, $p=0.006$ ) bone threshold limits. For example, for three patellae with highest $\rho(0.57 \pm 0.02)$, the bone volume with isotropic models was $2.5 \pm 0.4$ and $1.9 \pm 0.1$ times higher for $5 \%$ and $10 \%$ bone threshold limits, respectively, while for three patellae with lowest $\rho(0.30 \pm 0.03)$ the bone volume with isotropic models was $3.8 \pm 0.5$ and $2.7 \pm 0.6$ times higher for $5 \%$ and $10 \%$ threshold limits, respectively, while for the resurfaced case, the trend was similar, but the correlation was weaker and not significant.

\section{Discussion}

To assess the importance of anisotropy in predictions of patellar strain and to estimate the effect of the isotropic assumption, we compared strain predictions of 17 patellofemoral models of TKA assigned validated isotropic and anisotropic material. We considered both resurfaced and non-resurfaced patella. Our results confirmed the expected strain overestimation of the isotropic model.

The patellar bone has a high degree of anisotropy, with a trabecular architecture adapted to its physiological loading: inferosuperior tension in the anterior part and antero-posterior compression in the posterior part [7]. The overestimation of strain by the isotropic model is probably caused by the loss of stiffness along the main trabecular anisotropic direction. The highest impact was noticed in non-resurfaced rather than resurfaced patella. This is probably explained by the replacing of an important volume of anisotropic bone with the isotropic polyethylene material. Probably, less bone removal with resurfaced patellae will cause higher differences between isotropic and anisotropic models. The influence of anisotropy was more critical in low density non-resurfaced patellae. These observations are consistent with a similar study conducted on the proximal femur [9].

Several limitations of the study should be mentioned. The clinical reality and patient variability was simplified by one loading condition. We have chosen 60 degrees of knee flexion since this angle is associated with high mechanical loading, and is often reached during daily activities [19]. However, due to the trabecular structure of the patella, we do not expect important changes of our results and conclusions for different angles of flexion. The patella is expected to be loaded along the main trabecular direction during all flexion range. However, this assumption should be checked. The cortex of the patella was not explicitly modeled. To evaluate its effect, we compared model predictions, without and with cortex, on two non-resurfaced patellae: the one with highest average density and one with the lowest. The cortex was model by isotropic and homogeneous shell elements ( $E=12 \mathrm{GPa}, v=0.3$, thickness $=0.5 \mathrm{~mm}$ ) [20]. The lack of the thin cortex layer indeed weakens the patella and increases strains, especially for low density patellae. However, these limitations of the present model had a slight influence on the relative difference between isotropic and anisotropic model predictions, and thus did not affect our conclusion. We considered specific strain thresholds for each patella due to unique boundary conditions (muscle forces). Adaptation of boundary conditions of each model to the height and weight of 
the patellar donor and considering only one strain threshold (e.g., approximate level of bone yield) is expected to increase the correlation between the average bone volume fraction and effect caused by isotropic assumption, but probably will not change the order of the average effect, as our conclusions. The reported correlation between the bone volume fraction and the difference in the isotropic and anisotropic strain volumes might be affected by the size and shape of the patella, as well as the position of the prosthetic component. Inclusion of these factors would probably improve the correlation, but we still assume that bone volume fraction has the highest influence.

To conclude, not accounting for anisotropy can drastically and significantly overestimate prediction of patellar strain, especially for patellae with a low bone volume fraction. This finding is essential for patient-specific modeling of patellar strain. Because of challenges to obtain patellar anisotropy from standard preoperative CT scans [8], the isotropic assumption remains a practical limitation in patient-specific clinical applications. Moreover, patellar bone quality of TKA patients is often low, likely because of preoperative and postoperative reduced daily motion due to pain, and, additionally, may vary between patients of different pain level $[21,22]$. Therefore, patellar isotropic models should be used with caution and account for possible inaccuracy. Alternatively, when bone anisotropy cannot be measured directly, a generic or statistical map of anisotropy could be applied to the patella [23] or it can be obtained with template registration approach [24].

\section{Conflict of interest}

None of the authors has any conflict of interest.

\section{Funding}

This project was funded by the "Fondation de soutien a'la recherche dans le domaine de l'orthopedie-traumatologie".

\section{Ethical approval}

This study was performed on existing micro-CT data of cadaveric patellae. Approval was obtained from National Disease Researcher Interchange (NDRI) to obtain these cadaveric samples. Local ethical committee approval was not required.

\section{Acknowledgments}

This project was partly funded by the "Fondation de soutien a'la recherche dans le domaine de l'orthopedie-traumatologie".

\section{References}

[1] Chun KA, Ohashi K, Bennett DL, El-Khoury GY. Patellar fractures after total knee replacement. AJR Am J Roentgenol 2005;185:655-60.
[2] Schindler OS. The controversy of patellar resurfacing in total knee arthroplasty: ibisne in medio tutissimus? Knee Surg Sports Traumatol Arthrosc Off J ESSKA 2012:20:1227-44.

[3] Fitzpatrick CK, Baldwin MA, Ali AA, Laz PJ, Rullkoetter PJ. Comparison of patellar bone strain in the natural and implanted knee during simulated deep flexion. J Orthop Res 2011;29(2):232-9.

[4] Fitzpatrick CK, Kim RH, Ali AA, Smoger LM, Rullkoetter PJ. Effects of resection thickness on mechanics of resurfaced patellae. J Biomech 2013;46:1568-75.

[5] Ho KY, Keyak JH, Powers CM. Comparison of patella bone strain between females with and without patellofemoral pain: a finite element analysis study. J Biomech 2014;47:230-6.

[6] Takahashi A, Sano H, Ohnuma M, Kashiwaba M, Chiba D, Kamimura M, et al. Patellar morphology and femoral component geometry influence patellofemoral contact stress in total knee arthroplasty without patellar resurfacing. Knee Surg Sports Traumatol Arthrosc Off J ESSKA 2012;20:1787-95.

[7] Latypova A, Maquer G, Elankumaran K, Pahr D, Zysset P, Pioletti DP, et al. Identification of elastic properties of human patellae using micro-finite element analysis. J Biomech 2016;49:3111-15.

[8] Nazemi SM, Cooper DM, Johnston JD. Quantifying trabecular bone material anisotropy and orientation using low resolution clinical CT images: a feasibility study. Med Eng Phys 2016;38:978-87.

[9] Hazrati Marangalou J, Ito K, van Rietbergen B. A new approach to determine the accuracy of morphology-elasticity relationships in continuum FE analyses of human proximal femur. J Biomech 2012;45:2884-92.

[10] Latypova A, Arami A, Becce F, Jolles-Haeberli B, Aminian K, Pioletti DP, et al. A patient-specific model of total knee arthroplasty to estimate patellar strain: a case study. Clin Biomech 2016;32:212-19.

[11] Cutts A, Seedhom BB. Validity of cadaveric data for muscle physiological crosssectional area ratios: a comparative study of cadaveric and in-vivo data in human thigh muscles. Clin Biomech 1993;8:156-62.

[12] Zysset PK. A review of morphology-elasticity relationships in human trabecular bone: theories and experiments. J Biomech 2003;36:1469-85.

[13] Zysset PK, Curnier A. An alternative model for anisotropic elasticity based on fabric tensors. Mech Mater 1995;21:243-50.

[14] Cowin SC. The relationship between the elasticity tensor and the fabric tensor. Mech Mater 1985;4:137-47.

[15] Moglo KE, Shirazi-Adl A. On the coupling between anterior and posterior cruciate ligaments, and knee joint response under anterior femoral drawer in flexion: a finite element study. Clin Biomech 2003;18:751-9.

[16] Pahr DH, Zysset PK. A comparison of enhanced continuum FE with micro FE models of human vertebral bodies. J Biomech 2009;42:455-62.

[17] Abaqus. Abaqus v 6.12 User Documentation. Dessault Systems. 2012

[18] Kettenberger U, Latypova A, Terrier A, Pioletti DP. Time course of bone screw fixation following a local delivery of Zoledronate in a rat femoral model - a micro-finite element analysis. J Mech Behav Biomed Mater 2015;45:22-31.

[19] Mason JJ, Leszko F, Johnson T, Komistek RD. Patellofemoral joint forces. J Biomech 2008;41:2337-48.

[20] Eckstein F, Milz S, Anetzberger H, Putz R. Thickness of the subchondral mineralised tissue zone (SMZ) in normal male and female and pathological human patellae. J Anat 1998;192(1):81-90.

[21] Di Martino A, Franceschi F, Papalia R, Marini M, Prossomariti G, Maffulli N, et al. Increased bone mineral density in the non-resurfaced patella after total knee arthroplasty: a clinical and densitometric study. Surg J R Coll Surg Edinb Irel 2012;10:20-4.

[22] Burnett W, Kontulainen S, McLennan C, Hazel D, Talmo C, Hunter D, et al. Patella bone density is lower in knee osteoarthritis patients experiencing moderate-to-severe pain at rest. J Musculoskelet Neuronal Interact 2016;16:33-9.

[23] Marangalou JH, Ito K, Cataldi M, Taddei F, van Rietbergen B. A novel approach to estimate trabecular bone anisotropy using a database approach. J Biomech 2013;46:2356-62.

[24] Taghizadeh E, Reyes M, Zysset P, Latypova A, Terrier A, Büchler P. Biomechanical role of bone anisotropy estimated on clinical CT scans by image registration. Ann Biomed Eng 2016;44(8):2505-17. 